\title{
Autonomic dysfunction in patients with COVID-19
}

\author{
Yuksel Erdal $^{1}{ }^{\mathbb{D}} \cdot$ Arife Cimen Atalar $^{1} \cdot$ Taskın Gunes $^{2} \cdot$ Tugba Okluoglu $^{1} \cdot$ Nurettin Yavuz $^{1} \cdot$ Ufuk Emre $^{1}$
}

Received: 18 September 2021 / Accepted: 17 February 2022 / Published online: 3 March 2022

(C) The Author(s) under exclusive licence to Belgian Neurological Society 2022

\begin{abstract}
Purpose Autonomic dysfunction in patients with viral infections has been described before. In this study, we aimed to evaluate autonomic functions in patients with the coronavirus infectious disease 2019 (COVID-19).

Methods In this cross-sectional study, we compared 112 patients who had recovered from COVID-19 and 106 healthy controls. Symptoms of autonomic dysfunction were assessed with the SCOPA-AUT scale.

Results Pupillomotor, urinary and sudomotor subscores of SCOPA-AUT scale were significantly higher in the COVID-19 patient group ( $p=0.03, p=0,006, p=0.0001$, respectively). There were no significant difference in terms of gastrointestinal, cardiovascular, sexual subscores and total SCOPA-AUT scores between the patient and control groups. The presence of fatigue symptom in the acute phase of COVID-19 increased the total SCOPA-AUT score by 2.2 points $(p=0.04)$ whereas the presence of smell loss $(\mathrm{OR}=5.82, p=0.01)$ and dyspnea $(\mathrm{OR}=5.8, p=0.03)$ were significant risk factors for pupillomotor dysfunction. The urinary, cardiovascular, sexual subscores and the total score of SCOPA-AUT scale were positively correlated with the age of the patient group.

Conclusion Our study suggests that many patients might have prolonged symptoms of autonomic dysfunction after the acute phase of COVID-19 that might worsen the clinical recovery.
\end{abstract}

Keywords COVID-19 $\cdot$ Autonomic dysfunction $\cdot$ SCOPA-AUT $\cdot$ Sudomotor

\section{Introduction}

The coronavirus infectious disease 2019 (COVID-19), caused by novel severe acute respiratory syndrome coronavirus 2 (SARS-CoV-2), rapidly spread throughout the world leading to a pandemic disaster, and causing a serious mortality and morbidity especially for risky groups [1]. Although the most common clinical features of the disease are fever, dry cough and dyspnea, neurological manifestations are also very common and even might be the initial symptoms of the disease [2, 3]. In non-severe COVID-19 patients, the presented neurological symptoms are similar to the ones in other common respiratory virüs infections (etc.; influenza) such as fatigue, headache, vertigo and nonspecific symptoms like anosmia and

Yuksel Erdal

erdalyuksel_355@hotmail.com

1 Department of Neurology, Health Sciences University, Istanbul Training and Research Hospital, Istanbul, Turkey

2 Department of Neurology, Bahcesehir University, VM Maltepe Medical Park Hospital, Istanbul, Turkey ageusia. A proportion of critically ill patients develop central nervous system involvement such as encephalitis, encephalomyelitis, ischemic stroke and peripheral nervous system disorders like Guillain-Barre syndrome that might cause additional complications for the patients suffering from COVID-19 [4-7]. Although evidence of dysautonomia in COVID-19 infection is limited, a body of data is accumulating and studies focusing on autonomic system involvement suggest that, autonomic dysfunction is not a rare comorbidity in these patients and might exacerbate COVID-19 mortality [7].

Patients with autonomic nervous system dysfunction can present with a wide-range of symptoms including abnormalities in heart rate, blood pressure, thermoregulation, sexual functions, and bowel and bladder functions $[8,9]$. Autonomic dysfunction have been reported in many of the viral infections, such as retroviruses (human immune deficiency virus (HIV)), herpes viruses, flavivirus, enterovirus and lyssavirus [10]. The 2009 pandemic of Influenza A (H1N1) is one of these viruses that have been reported to cause cardiac autonomic dysfunction in infants older than 1 year of age. [11]. Although the pathophysiological 
mechanisms underlying the autonomic system involvement in viral infections are scarce, direct invasion of the central nervous system, peripheral nervous system invasion and immune-mediated disorders are some of the proposed mechanisms $[10,12]$.

Autonomic dysfunction in cases of Guillain Barre syndrome related to COVID-19 [13] and acute motor axonal neuropathy (AMAN) presenting with dysautonomia symptoms were reported before [14]. Postural tachycardia syndrome (POTS) were also observed as a postinfectious complication of COVID-19 [15-17]. The American Autonomic Association has released an extensive paper recently, implying the importance of autonomic functioning and recommended further testing in patients with COVID-19 [18], but there is still a gap in this area and prospective studies are needed to further investigate the spectrum of autonomic dysfunction related to COVID-19. In this study, we aimed to evaluate the autonomic functions of patients recovered from COVID-19 at the post-infectious stage of the disease.

\section{Methods}

We enrolled 112 patients who had recovered from COVID19 and 106 healthy controls in this study. Patients were recruited randomly from the neurology outpatient clinics, in Istanbul Training and Research Hospital between January and April 2021. For all patients, COVID-19 infection was confirmed from RT-PCR and/or antigen test reports. Demographic characteristics of the patients and clinical symptoms in the period following RT-PCR positivity were recorded. Patients with confirmed diagnoses of neurodegenerative diseases, polyneuropathy, metabolic diseases, heart diseases, using drugs with significant anticholinergic effects, chronic alcoholism and daily smokers were excluded from the study. Autonomic functions were measured using the SCOPA-AUT scale.

106 healthy individuals without clinical evidence of either previous or current COVID-19 were included as controls. The control group was randomly selected among the people and their relatives who applied to the neurology outpatient clinic and exclusion criteria were also similar for the control group. Local ethics committee approved the study (Decision number: 2578, Date: 13.11.2020). Written informed consent was obtained from all participants.

\section{The SCOPA-AUT scale}

The SCOPA-AUT scale determines the presence and frequency of autonomic symptoms [19]. The scale consists of a total of 23 items with the following 6 domains; gastrointestinal (7 items), urinary (6 items), cardiovascular (3 items), thermoregulator (4 items), pupillomotor (1 item), and sexual
( 2 items for males and 2 items for females) dimensions. Responses for the frequency of each item are scored between 0 (never) and 3 (often), higher scores reflecting more severe autonomic dysfunction. The highest possible score is 99. All questions are based on experiences in the last month while the syncope query is based on the last 6 months. The Turkish validity and reliability study of the SCOPA-AUT scale was conducted by Yasar et al. [20].

\section{Statistical analysis}

Descriptive statistics are presented as frequencies, medians (with range), means (with standard deviations) or proportions, as appropriate. Normality was tested using $Q-Q$ plot and histogram. Continuous variables were analysed with independent samples $t$-test and Mann- $U$ Whitney tests. Categorical variables were analysed by chi-square test. The relationship between acute clinical symptoms of COVID19 and the final SCOPA-AUT score were further analysed by linear logistic regression analysis. The association of autonomic functions with disease duration and age were evaluated by Spearmann correlation analysis. All statistical analysis were performed using Stata12 (Stata Corp LP, Texas, USA) software programme. A $p$ value $\leq 0.05$ was considered significant.

\section{Results}

Gender distribution of the patient and control groups were similar (F/M, 68/44 vs 64/42, $p=0.9)$. The mean age of the control group was higher than the patient group $(45.7 \pm 12.5$ and $40.3 \pm 12.5$, respectively $(p=0.001)$.

Ten patients $(9 \%)$ were hospitalized at the acute phase of COVID-19 infection. The time duration between the COVID-19 PCR test positivity and the application of the SCOPA-AUT test to the patients was $3.7 \pm 1.7$ (min-max 1-9) months. The most common symptom was fatigue (73.2\%) at the acute phase of COVID-19. The distribution of the clinical symptoms of patients at the acute phase are summarized in Table 1.

The pupillomotor, urinary and sudomotor subscores of SCOPA-AUT scale of the patients were statistically higher than the control group $(p=0.03, p=0,006$ and $p=0.001$, respectively). There were no significant difference in terms of gastrointestinal, cardiovascular and sexual subscores $(p>0.05)$. The total SCOPA-AUT scores were also similar between the patient and control groups $(p>0.05)$ (Table 2).

In the linear regression analysis, we found that the presence of fatigue increased the total SCOPA-AUT scores by 2.2 folds [OR:2.2, $p=0.04]$. With regard to SCOPA-AUT subscores, having anosmia $(\mathrm{OR}=5.82, p=0.01)$ and dyspnea $((\mathrm{OR}=5.8, p=0.03)$ could increase the pupillomotor 
dysfunction risk at the post COVID phase. The presence of other clinical symptoms at the acute phase of the infection had no significant effect over the SCOPA-AUT scores. In addition, there was no significant difference between the SCOPA-AUT scores of the hospitalized or non-hospitalized patients (Table 3).

When we performed a correlation analysis, we found a positive correlation between the urinary $(r=0.30$, $p=0.001)$, cardiovascular $(r=0.22, p=0.01)$ and sexual $(r=0.30, p=0.001)$ subscores and the total SCOPA-AUT

Table 1 Demographic and clinical characteristics of the COVID-19 patient and control groups

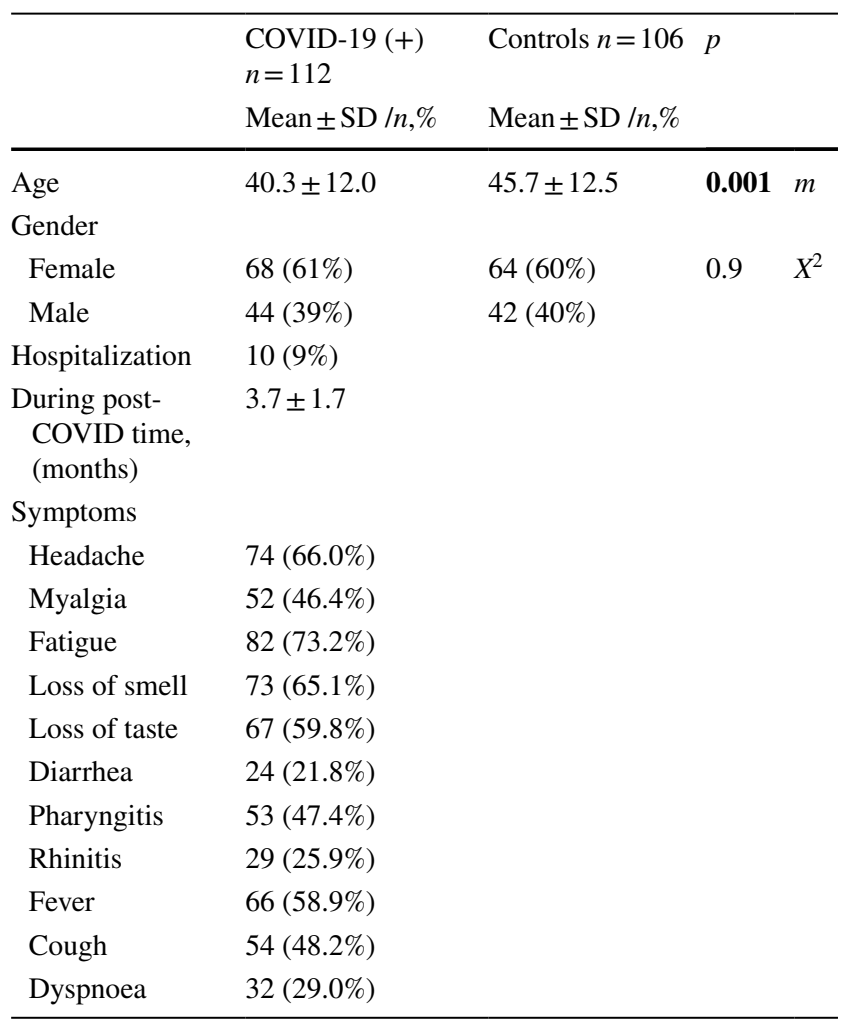

Boldface values indicate significant results $(p<0.05)$

$m$ Mann-Whitney $U$ test; $X^{2}$ Chi square test, scores $(r=0.27, p=0.03)$, with the age of the patients. There was no correlation between the time duration after COVID19 and SCOPA-AUT scores (Table 4).

\section{Discussion}

In this study, we found that symptoms of autonomic dysfunction are very common at the post-infectious period of COVID-19. The most common symptom at the acute phase was fatigue and the presence of fatigue increased the risk of autonomic dysfunction by 2.2 folds at the post-COVID period. Also, higher scores on SCOPA-AUT scale were found to be associated with older age. Our results could give clues for the management of autonomic symptoms at the post-infectious phase of COVID-19.

There are insufficient data to show that SARS-CoV-2 directly infects the brain [21]. However, serious neurological symptoms are seen in COVID-19 disease, one of which is autonomic dysfunction [7, 22]. There are several studies reporting the neurological system involvement in addition to the respiratory, cardiovascular and other systematic complications of the disease in the acute and later stages but data on the autonomic system involvement is still limited. We suggest that COVID-19 could lead to autonomic dysfunction symptoms at the post infectious stage of the disease in addition to its other neurological and systematical symptoms at the acute phase such as headache, anosmia, ageusia, myalgia and fatigue. We showed that sudomotor, pupillomotor and urinary dysfunction symptoms could emerge at the later stages which could complicate the recovery phase of the patient. We believe these autonomic dysfunction symptoms could be a part of the so called "long COVID" spectrum of the disease [23, 24].

Fatigue appears to be a very common presentation of the disease both at the acute and post infectious stages of COVID-19 [25]. It was reported to be directly related to autonomic dysfunction in some of the reported studies [26]
Table 2 Comparison of the SCOPA-AUT scale parameters between COVID-19 patients and controls

\begin{tabular}{|c|c|c|c|c|c|}
\hline & \multicolumn{2}{|c|}{ Patients $(n=112)$} & \multicolumn{2}{|c|}{ Controls $(n=106)$} & \multirow[t]{2}{*}{$p$} \\
\hline & Mean \pm SD & Median & Mean \pm SD & Median & \\
\hline \multicolumn{6}{|l|}{ SCOPA-AUT scale } \\
\hline Pupillomotor & $0.4 \pm 0.7$ & 0.3 & $0.6 \pm 0.7$ & 0.5 & 0.03 \\
\hline Sudomotor & $3.1 \pm 2.5$ & 3.0 & $1.7 \pm 1.8$ & 1.0 & 0.0001 \\
\hline Gastrointestinal & $2.9 \pm 2.1$ & 2.0 & $2.5 \pm 2.1$ & 2.0 & 0.7 \\
\hline Cardiovascular & $1.8 \pm 1.6$ & 2.0 & $1.9 \pm 2.4$ & 1.0 & 0.2 \\
\hline Urinary & $3.6 \pm 3.2$ & 3.0 & $2.5 \pm 2.2$ & 2.0 & 0.006 \\
\hline Sexual & $0.7 \pm 1.1$ & 0.6 & $0.9 \pm 1.2$ & 0.1 & 0.1 \\
\hline Total points & $12.7 \pm 9.6$ & 10.0 & $10.4 \pm 7.7$ & 8.0 & 0.06 \\
\hline
\end{tabular}

Boldface values indicate significant results $(p<0.05)$

$m$ Mann-Whitney u test, 
Table 3 Linear logistic regression analysis of association between acute symptoms of COVID-19 infection, post-COVID duration time and SCOPA-AUT parameters

\begin{tabular}{|c|c|c|c|c|c|c|c|}
\hline & Pupilomotor & Sudomotor & GIS & Cardiovascular & Urinary & Sexual & Total \\
\hline \multicolumn{8}{|c|}{ Headache } \\
\hline OR & 1.21 & 1.57 & 1.23 & 1.40 & 1.14 & 1.08 & 1.02 \\
\hline$p$ & 0.7 & 0.2 & 0.5 & 0.3 & 0.7 & 0.8 & 0.2 \\
\hline \multicolumn{8}{|c|}{ Myalgia } \\
\hline OR & 2.43 & 1.26 & 0.80 & 0.55 & 0.96 & 2.02 & 1.01 \\
\hline$p$ & 0.1 & 0.5 & 0.5 & 0.1 & 0.9 & 0.1 & 0.4 \\
\hline \multicolumn{8}{|c|}{ Fatigue } \\
\hline OR & 0.36 & 0.65 & 1.22 & 1.20 & 0.65 & 0.55 & 2.2 \\
\hline$p$ & 0.1 & 0.3 & 0.6 & 0.6 & 0.3 & 0.2 & 0.04 \\
\hline \multicolumn{8}{|c|}{ Loss of smell } \\
\hline OR & 5.82 & 1.56 & 1.44 & 2.96 & 0.61 & 1.38 & 1.02 \\
\hline$p$ & 0.01 & 0.4 & 0.5 & 0.08 & 0.4 & 0.6 & 0.2 \\
\hline \multicolumn{8}{|c|}{ Loss of taste } \\
\hline OR & 0.32 & 0.83 & 0.69 & 0.44 & 2.43 & 0.60 & 1.01 \\
\hline$p$ & 0.08 & 0.7 & 0.4 & 0.1 & 0.1 & 0.4 & 0.4 \\
\hline \multicolumn{8}{|c|}{ Dyspnea } \\
\hline OR & 3.05 & 1.84 & 1.98 & 2.00 & 1.08 & 1.68 & 1.01 \\
\hline$p$ & $\mathbf{0 . 0 3}$ & 0.1 & 0.09 & 0.1 & 0.8 & 0.2 & 0.5 \\
\hline \multicolumn{8}{|c|}{ Diarrhea } \\
\hline OR & 0.80 & 0.96 & 0.72 & 1.51 & 1.22 & 0.45 & 1.03 \\
\hline$p$ & 0.6 & 0.9 & 0.4 & 0.3 & 0.6 & 0.1 & 0.07 \\
\hline \multicolumn{8}{|c|}{ Duration (months) } \\
\hline OR & 1.3 & 1.5 & 0.9 & 1.1 & 1.0 & 0.6 & 1.03 \\
\hline$p$ & 0.4 & 0.4 & 0.9 & 0.5 & 0.3 & 0.3 & 0.9 \\
\hline
\end{tabular}

Boldface values indicate significant results $(p<0.05)$

$O R$ odds ratio
Table 4 Correlation analyses of patients with post-COVID-19 duration, age and SCOPA-AUT parameters

\begin{tabular}{|c|c|c|c|c|c|c|c|}
\hline & Pupillomotor & Sudomotor & GIS & Cardiovascular & Urinary & Sexual & $\begin{array}{l}\text { Total } \\
\text { MOCI } \\
\text { score }\end{array}$ \\
\hline \multicolumn{8}{|c|}{ Age } \\
\hline$r$ & 0.09 & 0.18 & 0.13 & 0.22 & 0.30 & 0.30 & 0.27 \\
\hline$p$ & 0.3 & 0.05 & 0.1 & 0.01 & 0.001 & 0.001 & 0.003 \\
\hline \multicolumn{8}{|c|}{ Duration (month) } \\
\hline$r$ & 0.12 & 0.12 & 0.10 & 0.12 & 0.05 & 0.14 & 0.10 \\
\hline$p$ & 0.2 & 0.1 & 0.3 & 0.2 & 0.6 & 0.1 & 0.3 \\
\hline
\end{tabular}

Spearman correlation

GIS gastrointestinal and the relationship of fatigue with autonomic dysfunction in some other neurological diseases such as multiple sclerosis [27], Parkinson's disease [28], sleep disorders [29] and chronic hepatitis [30] is also well known. Although Towsend et al. reported that there was no difference in terms of autonomic dysfunction symptoms between patients with fatigue and non-fatigue [31], our results show that the presence of fatigue at the acute stage of SARS-CoV-2 infection increases the risk of autonomic dysfunction by 2.2 folds at the post
COVID phase. Fatigue could be a risk factor for more severe neurological symptoms related to autonomic dysfunction at the later stages of the disease that will impair quality of life with an impact on the individuals' personal and professional life.

The mechanisms underlying the association between COVID-19 and autonomic dysfunction is still uncovered. The invasion of the virus and the inflammatory cytokines to the median eminencia of the hypothalamus, could result 
with the activation of the autonomic nervous system and the release of adrenal catecholamines and steroids [2]. Also, entry of the virus and/or proinflammatory molecules through the subfornical organ and the area postrema could also affect brainstem autonomic pathways that are controlling blood pressure and breathing [32]. Alternatively, COVID-19-related autonomic dysfunction could be mediated by the virus itself [33]. Another hypothesis is that an underlying autoimmune mechanism can be responsible from the infectionrelated autonomic dysfunction. In certain autonomic syndromes such as orthostatic hypotension $(\mathrm{OH})$ and POTS, the presence of autoantibodies against $\alpha$-/ $\beta$-adrenoceptors and muscarinic receptors is already shown [34]. The cytokine storming induced by the autonomic nervous system and neurohumoral effect could lead to end-organ damage at the older individuals with comorbid diseases which could result with a vicious circle that could be life-threatening [35].

In a recent observational study, 50 patients were evaluated with the National Health Service (NHS) questionnaire for neurological symptoms and electrochemical skin conductance test. Sudomotor dysfunction was shown in $26 \%$ of the patients revealing that sweat dysfunction reflecting dysautonomia is very common at the recovery phase of COVID-19 [36]. Similarly, in another retrospective study, Shouman et al. reported that $26 \%$ of their patients with COVID-19 showed sudomotor dysfunction symptoms [37]. There are also several case reports revealing the presence of sudomotor dysfunction after COVID-19 [38-40]. Peripheral nervous system involvement and related neurological symptoms in COVID-19 suggest that small demyelinated nerve fibers might be involved but the role of central nervous system (CNS) in autonomic dysfunction could not be ruled out. Unlike CNS, there is no evidence that the virus could directly invase the peripheral nervous system (PNS) but rather damage the PNS by a cytokine storming or dysimmune mechanism [36]. Stella et al. performed a questionnaire-based study on a large sample of long-COVID patients with and without neurological symptoms, using the COMPASS-31 questionnaire, and found that orthostatic intolerance, sudomotor, gastrointestinal and pupillomotor dysfunction were the most effected domains of the autonomic nervous system in these patients. In addition, COMPASS-31 scores were higher in patients with neurological symptoms due to more severe orthostatic intolerance whereas sudomotor, gastrointestinal and pupillomotor functions were more severely affected in patients without neurological symptoms [41]. In our study, the most significant difference was found in sudomotor subscore of SCOPA-AUT scale in the patient group comparing to the other subscores or the total score which suggest that there could be an underlying small fibre neuropathy. Small fibre neuropathies were reported after several viral infections before [42] but the causative relationship of the neuropathy and the viral infection is still unclear and yet to be proven. We believe further studies performed with quantitative autonomic testing methods in larger cohorts are needed to highlight the nature of autonomic dysfunction in these patients.

Autonomic nervous system have been traditionally composed of sympathetic nervous system, parasympathetic nervous system, and the enteric nervous system, however neuroendocrine and neuroimmune systems have recently been identified and expanded the concept of "autonomic" [43]. Hightened sympathetic nervous system activity accompanies chronic cardiovascular and endocrine diseases such as hypertension, cardiac failure, type 2 diabetes mellitus and chronic renal failure and contributes to the progression of these diseases [44]. Recent epidemiological studies report that COVID-19 patients with these comorbid diseases are more prone to mortal complications and mortality [35, 45].

Although we excluded patients with comorbid diseases such as diabetes mellitus, multiple sclerosis, neurodegenerative disease etc. that might have severe autonomic system involvement, we observed that our patients with COVID-19 had prominent autonomic dysfunction symptoms. Moreover, despite the older age of the control group, the presence of autonomic symptoms were lower than the patients supporting that COVID-19 might lead to autonomic dysfunction independent from the age of the patient.

Cardiovascular complications such as arrhythmia, myocarditis, cardiac failure, and myocardial infarction have been widely reported before. It is hypothesised that COVID-19 could induce sympathetic nervous system over activity and worsen the prognosis [46]. Recently American Autonomic Society provided a statement to guide physicians, health systems and researchers about the impact of long-COVID POTS which occurs when the commonly described symptoms of long-COVID are accompanied by excessive orthostatic tachycardia [47]. We did not have any significant difference in terms of cardiac parameters between patient and control groups which could be related to the exclusion of patients with severe metabolic and vascular comorbid diseases.

Our study has some limitations of note. First, we did not perform quantitative standardized autonomic function tests such as thermoregulatory sweat test, quantitative sudomotor axon reflex test and heart rate variability test to the patient and control groups. As a second limitation, our patients were referred from our neurology outpatient clinics which could be a selection bias for this study therefore further studies performed with larger cohorts are needed to support our results. Also, we assessed the autonomic functions with an extensive range (1-9 months), so that means that the patients were not uniformly evaluated at the same time.

In conclusion, COVID-19 is associated with a high burden of symptoms of autonomic dysfunction especially in sudomotor, urinary and pupillomotor systems. Autonomic dysfunction should be questioned as a part of the standard 
neurological examination alongside the other neurological symptoms. Early diagnosis of autonomic dysfunction in patients with COVID-19 may help planning the management and limit the progression of the disease. Further studies with larger populations are needed to support our findings.

Author contributions YE: Conceptualization; investigation; methodology; writing original draft. TO and NY: Methodology; resources; data curation; formal analysis. ACA: Writing-review and editing. TG: Data curation; formal analysis. UE: Data supervision and editing.

Funding None.

\section{Declarations}

Conflict of interest The authors declare that they have no conflict of interest.

\section{References}

1. Chan AT, Drew DA, Nguyen LH, Joshi AD, Ma W, Guo CG et al (2020) The Coronavirus Pandemic Epidemiology (COPE) Consortium: a call to action. Cancer Epidemiol Biomarkers Prev 29:1283-1289

2. Iadecola C, Anrather J, Kamel H (2020) Effects of COVID-19 on the nervous system. Cell 183:16-27

3. Jiang F, Deng L, Zhang L, Cai Y, Cheung CW, Xia ZJJGIM (2020) Review of the clinical characteristics of coronavirus disease 19 (COVID-19). J Gen Intern Med 35:1545-1549

4. Gklinos P (2020) Neurological manifestations of COVID-19: a review of what we know so far. J Neurol 267:2485-2489

5. Niazkar HR, Zibaee B, Nasimi A, Bahri N (2020) The neurological manifestations of COVID-19: a review article. Neurol Sci 41:1667-1671

6. Helms J, Kremer S, Merdji H, Clere-Jehl R, Schenck M, Kummerlen $C$ et al (2020) Neurologic features in severe SARSCoV-2 infection. N Engl J Med 382:2268-2270

7. Romero-Sánchez CM, Díaz-Maroto I, Fernández-Díaz E, Sánchez-Larsen Á, Layos-Romero A, García-García J et al (2020) Neurologic manifestations in hospitalized patients with COVID-19: The ALBACOVID registry. Neurology 95:1060-1070

8. Rafanelli M, Walsh K, Hamdan MH, Buyan-Dent L (2019) Autonomic dysfunction: diagnosis and management. Handb Clin Neurol 167:123-137

9. Xiong L, Leung TWH (2019) Autonomic dysfunction in neurological disorders. Aging (Albany NY) 11:1903-1904

10. Carod-Artal FJ (2018) Infectious diseases causing autonomic dysfunction. Clin Auton Res 28:67-81

11. Mattéi J, Teyssier G, Pichot V, Barthélémy JC, Achour E, Pillet S et al (2011) Autonomic dysfunction in 2009 pandemic influenza A (H1N1) virus-related infection: a pediatric comparative study. Auton Neurosci 162:77-83

12. Brizzi KT, Lyons JL (2014) Peripheral nervous system manifestations of infectious diseases. Neurohospitalist 4:230-240

13. Su XW, Palka SV, Rao RR, Chen FS, Brackney CR, Cambi F (2020) SARS-CoV-2-associated Guillain-Barré syndrome with dysautonomia. Muscle Nerve 62:48-49
14. Ghosh R, Roy D, Sengupta S, Benito-León J (2020) Autonomic dysfunction heralding acute motor axonal neuropathy in COVID-19. J Neurovirol 26:964-966

15. Miglis MG, Prieto T, Shaik R, Muppidi S, Sinn D-I, Jaradeh S (2020) A case report of postural tachycardia syndrome after COVID-19. Clin Auton Res 30:449-451

16. Kanjwal K, Jamal S, Kichloo A, Grubb BP (2020) New-onset postural orthostatic tachycardia syndrome following coronavirus disease 2019 infection. J Innov Card Rhythm Manag 11:4302-4304

17. Goodman BP, Khoury JA, Blair JE, Grill MF (2021) COVID-19 dysautonomia. Front Neurol 12:624968

18. Figueroa JJ, Cheshire WP, Claydon VE, Norcliffe-Kaufmann L, Peltier A, Singer W et al (2020) American Autonomic Society. Autonomic function testing in the COVID-19 pandemic: an American Autonomic Society position statement. Clin Auton Res 30:295-297

19. Visser M, Marinus J, Stiggelbout A, Van Hilten JJ (2004) Assessment of autonomic dysfunction in Parkinson's disease: the SCOPA-AUT. Mov Disord 19:1306-1312

20. Yasar H, Senol MG, Tasdemir S et al (2016) Validity and reliabilityof the Turkish version of the scales for outcomes in Parkinson'sdisease-autonomic questionnaire (SCOPA-AUT). Mov Disord 31(suppl 2):516-517

21. Thakur KT, Miller EH, Glendinning MD, Al-Dalahmah O, Banu MA, Boehme AK et al (2021) COVID-19 neuropathology at Columbia University Irving Medical Center/New York Presbyterian Hospital. Brain 144:2696-2708

22. Puccioni-Sohler M, Poton AR, Franklin M, Silva SJD, Brindeiro $\mathrm{R}$, Tanuri A (2020) Current evidence of neurological features, diagnosis, and neuropathogenesis associated with COVID-19. Rev Soc Bras Med Trop 53:e20200477

23. Greenhalgh T, Knight M, A'Court C, Buxton M, Husain L (2020) Management of post-acute covid-19 in primary care. BMJ 370:m3026

24. Nath A (2020) Long-Haul COVID. Neurology 95:559-560

25. Lo YL (2021) COVID-19, fatigue, and dysautonomia. J Med Virol 2021(93):1213

26. Newton JL, Okonkwo O, Sutcliffe K, Seth A, Shin J, Jones DE (2007) Symptoms of autonomic dysfunction in chronic fatigue syndrome. QJM 100:519-526

27. Cortez MM, Nagi Reddy SK, Goodman B, Carter JL, Wingerchuk DM (2015) Autonomic symptom burden is associated with MS-related fatigue and quality of life. Mult Scler Relat Disord $4: 258-263$

28. Chou KL, Gilman S, Bohnen NI (2017) Association between autonomic dysfunction and fatigue in Parkinson disease. J Neurol Sci 15(377):190-192

29. Tanaka M, Tajima S, Mizuno K, Ishii A, Konishi Y, Miike T (2015) Frontier studies on fatigue, autonomic nerve dysfunction, and sleep-rhythm disorder. J Physiol Sci 65:483-498

30. Wang H, Zhou Y, Yan R, Ru GQ, Yu LL, Yao J (2019) Fatigue in chronic hepatitis B patients is significant and associates with autonomic dysfunction. Health Qual Life Outcomes 25(17):130

31. Townsend L, Moloney D, Finucane C, McCarthy K, Bergin C, Bannan C (2021) Fatigue following COVID-19 infection is not associated with autonomic dysfunction. PLoS ONE 25(16): 0247280

32. Kaur C, Ling EA (2017) The circumventricular organs. Histol Histopathol 32(9):879-892

33. Guilmot A, Maldonado Slootjes S, Sellimi A, Bronchain M, Hanseeuw B, Belkhir L et al (2021) Immune-mediated neurological syndromes in SARS-CoV-2-infected patients. J Neurol 268:751-757 
34. Dani M, Dirksen A, Taraborrelli P, Torocastro M, Panagopoulos D, Sutton R et al (2021) Autonomic dysfunction in "long COVID": rationale, physiology and management strategies. Clin Med (Lond) 21:63-67

35. Porzionato A, Emmi A, Barbon S, Boscolo-Berto R, Stecco C, Stocco E et al (2020) Sympathetic activation: a potential link between comorbidities and COVID-19. FEBS J 287:3681-3688

36. Hinduja A, Moutairou A, Calvet JH (2021) Sudomotor dysfunction in patients recovered from COVID-19. Neurophysiol Clin 51:193-196

37. Shouman K, Vanichkachorn G, Cheshire WP, Suarez MD, Shelly S, Lamotte GJ et al (2021) Autonomic dysfunction following COVID-19 infection: an early experience. Clin Auton Res $16: 1-10$

38. Umapathi T, Poh MQW, Fan BE, Li KFC, George J, Tan JYL (2020) Acute hyperhidrosis and postural tachycardia in a COVID19 patient. Clin Auton Res 30:571-573

39. Novak P (2020) Post COVID-19 syndrome associated with orthostatic cerebral hypoperfusion syndrome, small fiber neuropathy and benefit of immunotherapy: a case report. eNeurol Sci 21:1002

40. Lamotte G, Benarroch EE, Coon EA (2021) Paroxysmal hypothermia and hyperhidrosis with exacerbation after COVID-19 Infection. Clin Auton Res 31:327-329

41. Buoite Stella A, Furlanis G, Frezza NA, Valentinotti R, Ajcevic M, Manganotti P (2021) Autonomic dysfunction in post-COVID patients with and witfhout neurological symptoms: a prospective multidomain observational study. J Neurol 12:1-10

42. Hehir MK 2nd, Logigian EL (2014) Infectious neuropathies. Continuum (Minneap Minn) 20:1274-1292

43. Goldstein DS (2020) The extended autonomic system, dyshomeostasis, and COVID-19. Clin Auton Res 30:299-315

44. Andersen ST, Witte DR, Fleischer J, Andersen H, Lauritzen T, Jørgensen ME et al (2018) Risk factors for the presence and progression of cardiovascular autonomic neuropathy in type 2 diabetes: ADDITION-Denmark. Diabetes Care 41:2586-2594

45. Del Rio R, Marcus NJ, Inestrosa NC (2020) Potential role of autonomic dysfunction in Covid-19 morbidity and mortality. Front Physiol 11:561749

46. Matsushita K, Marchandot B, Jesel L, Ohlmann P, Morel O (2020) Impact of COVID-19 on the cardiovascular system: a review. J Clin Med 9:1407

47. Raj SR, Arnold AC, Barboi A, Claydon VE, Limberg JK, Lucci VM et al (2021) Long-COVID postural tachycardia syndrome: an american autonomic society statement. Clin Auton Res $31: 365-368$

Publisher's Note Springer Nature remains neutral with regard to jurisdictional claims in published maps and institutional affiliations. 